NEUTROPHILS

\section{Neutrophil differentiation is autophagy dependent}

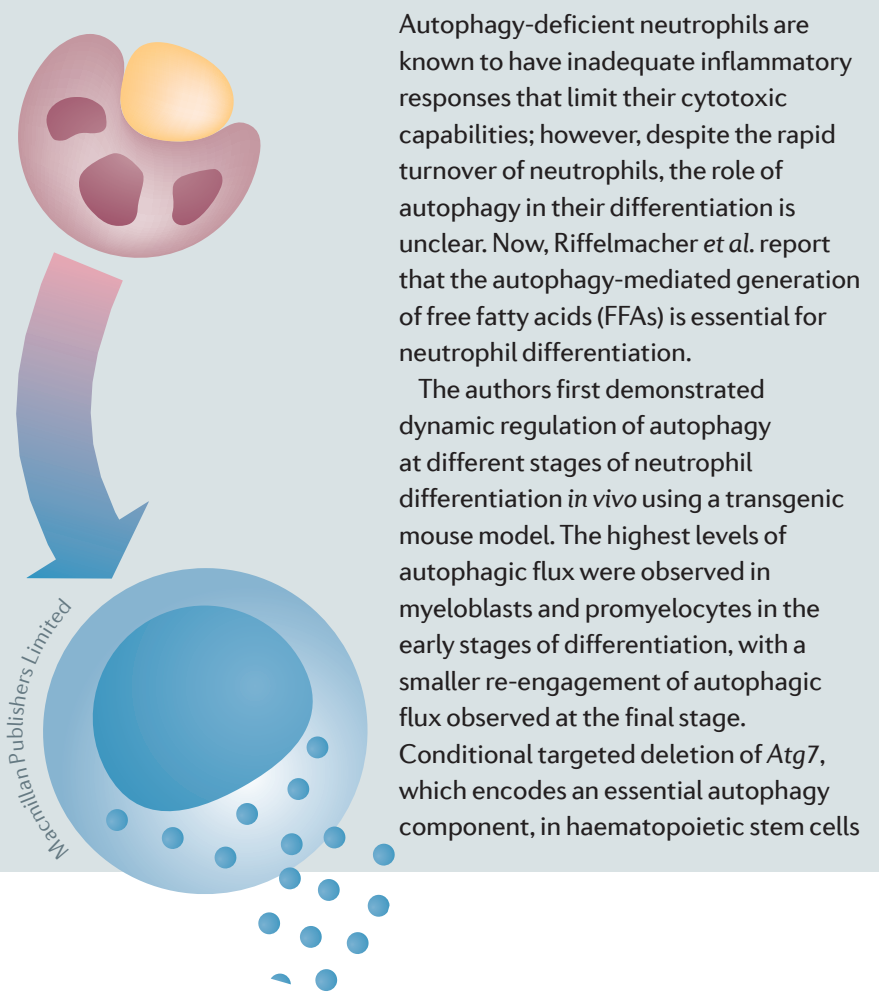

(HSCs) and progenitor cells leads to the accumulation of immature neutrophils, thus confirming the importance of autophagy. The authors then generated a mouse model with a more-targeted deletion of Atg7 in neutrophil progenitors, but not in HSCs. This model demonstrated a similar accumulation of immature neutrophils. Further evidence that this reflects deficient cell-intrinsic autophagy, and not other alterations during development, was provided by inducible deletion of Atg5, which is also critical to cell-intrinsic autophagy, in adult mice and in in vitro experiments.

The authors then examined alterations in metabolic requirements during neutrophil maturation. Metabolomic analysis of neutrophils undergoing granulocyte colony-stimulating factor-induced differentiation demonstrated a shift towards engagement of mitochondrial respiration, with downregulation of all glycolysis-associated genes. The same analysis of Atg7-deficient cells revealed upregulation of glycolysis at all stages of differentiation, suggesting that autophagy is required to engage mitochondrial respiration while also constraining glycolysis.

The authors also observed a depletion in FFA levels in Atg7-deficient myeloblasts in vitro relative to wild-type myeloblasts; similarly, ex vivo Atg7-deficient and Atg5-deficient neutrophils both showed signs of intracellular lipid accumulation, suggesting defective lipolysis.

Hypothesizing that oxidation of FFAs provides the necessary ATP for neutrophil differentiation, the authors were able to pharmacologically target this metabolic dependency to inhibit neutrophil maturation in wild-type myeloblasts. Furthermore, exposing Atg7-deficient neutrophil precursors to exogenous FFAs restored differentiation.

In summary, this research reveals the importance of cell-intrinsic autophagy in fulfilling the metabolic demands of neutrophil differentiation. These findings could guide the design of novel treatments for myeloid leukaemias or neutropenia.

Peter Sidaway

ORIGINAL ARTICLE Riffelmacher, T. et al. Autophagy-dependent generation of free fatty acids is critical for normal neutrophil differentiation. Immunity 47, 466-480 (2017) 